\title{
EDITORIAL
}

\section{Proceedings of the 11th Gravitational Wave Data Analysis Workshop, Potsdam, Germany, 18-21 December 2006}

The 11th Gravitational Wave Data Analysis Workshop (GWDAW11) comes in the middle of the fifth science run (S5) of the LIGO detectors, after a full year of data-taking at design sensitivity. LIGO is starting to probe astrophysically plausible scenarios for the emission of detectable gravitational wave signals. In the absence of a detection, the upper limits on the strength of gravitational waves are beginning to add information to what was previously known from electromagnetic observations.

One of the highlights of this workshop was the presentation by the LIGO Scientific Collaboration of a search for continuous waves from the Crab pulsar. Gravitational wave emission is constrained by the measured spindown measurements for the object: no more energy can go into gravitational waves than that which is lost by pulsar spindown. For most known pulsars this spindown upper limit on the strength of gravitational radiation is orders of magnitude higher than the upper limits that can be set with current gravitational wave observations. However for the Crab pulsar the gravitational wave observations which coherently combine about 9 months of data from the network of S5 LIGO detectors constrain the gravitational wave emission more strongly than the electromagnetic observations do. The gravitational wave upper limit also constrains the tri-axial ellipticity that this young pulsar could have supported since its crust solidified a thousand years ago.

The Virgo detector is in the final stages of commissioning with several engineering runs completed. The TAMA detector's low frequency performance is being enhanced and another data run is foreseen by the end of 2007. The IGEC2 Collaboration presents results from the analysis of 131 days of data in coincidence among the three bar detectors EXPLORER, AURIGA and NAUTILUS. Studies are underway to analyze data in coincidence among bar and interferometer networks.

Space-based detectors are receiving more attention, with the LISA Pathfinder mission planned for launch in 2010 and LISA in 2017. The special difficulties associated with data analysis for LISA are being addressed through the competitive mechanism of Mock LISA Data Challenges, and the results of the first challenge were presented and discussed at this workshop.

The different sessions and the presentations at GWDAW11 reflect this incredible richness of activity. This volume collects papers which cover many of the topics and work presented at GWDAW11. The complete set of presentation slides can be found at the conference site http://gwdaw11.aei.mpg.de/program.html.

As organizers of this workshop we would like to thank all the participants for taking part in this event and for the many lively discussions that we have enjoyed. We would also like to thank the editorial staff at CQG, especially Judith Adams and Tom Spicer, for their support and efficiency in preparing this volume.

B Krishnan, M A Papa and B F Schutz

Max Planck Institute for Gravitational Physics, Potsdam, Germany

\section{Guest Editors}

